\title{
Electroconvulsive Therapy Induces Cortical Morphological Alterations in Major Depressive Disorder Revealed with Surface-Based Morphometry Analysis
}

\author{
Jinping Xu \\ Institute of Biomedical and Health Engineering \\ Shenzhen Institutes of Advanced Technology \\ Chinese Academy of Sciences, Shenzhen 518055, P. R. China \\ University of Chinese Academy of Sciences \\ Beijing 100049, P. R. China \\ jp.xu@siat.ac.cn \\ Jiaojian Wang \\ The Clinical Hospital of Chengdu Brain Science Institute \\ MOE Key Lab for Neuroinformation \\ University of Electronic Science \\ and Technology of China, Chengdu 625014, P. R. China \\ jiaojianwang@uestc.edu.cn \\ Tongjian Bai \\ Department of Neurology, The First Hospital \\ of Anhui Medical University, Hefei 230022, P. R. China \\ Xiaodong Zhang and Tian Li \\ Institute of Biomedical and Health Engineering \\ Shenzhen Institutes of Advanced Technology \\ Chinese Academy of Sciences, Shenzhen 518055, P. R. China \\ Qingmao $\mathrm{Hu}$ \\ Institute of Biomedical and Health Engineering \\ Shenzhen Institutes of Advanced Technology \\ Chinese Academy of Sciences, Shenzhen 518055, P. R. China \\ CAS Key Laboratory of Human-Machine Intelligence-Synergy Systems \\ Shenzhen Institutes of Advanced Technology \\ Chinese Academy of Sciences, Shenzhen 518055, P. R. China \\ qm.hu@siat.ac.cn \\ Hongming $\mathrm{Li}$ \\ Center for Biomedical Image Computing and Analytics \\ Department of Radiology, Perelman School of Medicine \\ University of Pennsylvania, Philadelphia, PA 19104, USA \\ Li Zhang \\ Anhui Mental Health Center, Hefei 230022, P. R. China
}

This is an Open Access article published by World Scientific Publishing Company. It is distributed under the terms of the Creative Commons Attribution 4.0 (CC-BY) License. Further distribution of this work is permitted, provided the original work is properly cited. 


\author{
Qiang Wei \\ Department of Neurology \\ The First Hospital of Anhui Medical University \\ Hefei 230022, P. R. China \\ weiqiang19890914@126.com \\ Yanghua Tian \\ Department of Neurology \\ The First Hospital of Anhui Medical University \\ Hefei 230022, P. R. China \\ Department of Neurology \\ Shannan People's Hospital, Shannan, 856000, P. R. China \\ Kai Wang \\ Department of Neurology \\ The First Hospital of Anhui Medical University \\ Hefei 230022, P. R. China \\ Department of Medical Psychology \\ Anhui Medical University, Hefei 230022, P. R. China \\ Anhui Province Key Laboratory of Cognition and Neuropsychiatric Disorders \\ Hefei 230022, P. R. China \\ Collaborative Innovation Center for Neuropsychiatric Disorders and Mental Health \\ Hefei 230022, P. R. China
}

Accepted 21 January 2019

Published Online 12 March 2019

\begin{abstract}
Although electroconvulsive therapy (ECT) is one of the most effective treatments for major depressive disorder (MDD), the mechanism underlying the therapeutic efficacy and side effects of ECT remains poorly understood. Here, we investigated alterations in the cortical morphological measurements including cortical thickness (CT), surface area (SA), and local gyrification index (LGI) in 23 MDD patients before and after ECT. Furthermore, multivariate pattern analysis using linear support vector machine (SVM) was applied to investigate whether the changed morphological measurements can be effective indicators for therapeutic efficacy of ECT. Surface-based morphometry (SBM) analysis found significantly increased vertex-wise and regional cortical thickness (CT) and surface area (SA) in widespread regions, mainly located in the left insula (INS) and left fusiform gyrus, as well as hypergyrification in the left middle temporal gyrus (MTG) in MDD patients after ECT. Partial correlational analyses identified associations between the morphological properties and depressive symptom scores and impaired memory scores. Moreover, SVM result showed that the changed morphological measurements were effective to classify the MDD patients before and after ECT. Our findings suggested that ECT may enhance cortical neuroplasticity to facilitate neurogenesis to remit depressive symptoms and to impair delayed memory. These findings indicated that the cortical morphometry is a good index for therapeutic efficacy of ECT.
\end{abstract}

Keywords: Major depressive disorder; electroconvulsive therapy; cortical thickness; surface area; local gyrification index.

\section{Introduction}

Electroconvulsive therapy (ECT) has been reported to be one of the most effective treatments with rapid action for major depressive disorder (MDD)! 1 However, the widespread use of ECT is limited by its cognitive side effects, i.e. memory impairment! ${ }^{2}$ Although ECT has been used in clinical practice for decades, the mechanism underlying the therapeutic efficacy and side effects of ECT in MDD patients remains poorly understood. Most evidence from animal models supported the neurotrophic hypothesis that the effect of ECT is partially mediated by seizure-induced neurotrophic effects, resulting in increased rates of neurogenesis, synaptogenesis and glial proliferation ${ }^{3+6}$ In addition, the neurotrophic effects may contribute to the antidepressant effect ${ }^{9}$ 
and cognitive side effects! 78 These neurotrophic effects of ECT were detectable not only at the molecular and microstructural levels but also at the macroscopic level associated with brain structural changes.

To investigate the brain structural changes induced by ECT in MDD patients, a number of studies were performed using voxel-based morphometry (VBM), reporting changes in gray matter volume in various brain regions, such as the hippocampus, 12 amygdala,$\frac{13}{15}$ and temporal lobe.$^{16 / 17}$ However, a recent study using VBM did not identify changes in gray matter volumes in MDD patients after ECT even though the clinical symptoms were significantly improved ${ }^{[9}$ Given that the measurement of gray matter volumes using VBM includes information of cortical thickness (CT), surface area (SA) and cortical folding, 18 delineating the exact changes in $\mathrm{CT}, \mathrm{SA}$ and cortical folding in MDD patients after ECT will provide important insight to understand the mechanism of ECT.

Surface-based morphometry (SBM) analyses provide a way to directly measure $\mathrm{CT}, \mathrm{SA}$ and cortical folding of the brain in vivo. These three measurements provide specific parameters that can reflect distinct developmental trajectories of cortical structures and are known to be determined by distinct biological factors. ${ }^{1920}$ Specially, CT might be associated with neuronal density ${ }^{21 / 22}$ Local SA mainly reflects the number and spacing of cortical columns in a cortical regior ${ }^{23}$ and is suggested to be driven by cellular events such as synaptogenesis, gliogenesis and intracortical myelination.24 Cortical folding defined by the local gyrification index (LGI) allows a larger cortical SA (there are a greater number of neurons) to fit in the skull. Cortical folding may be related to the developmental integrity of cortical circuits, 25 providing forces driving the extensive wiring of cortico-cortical connections along the brain surface. ${ }^{26]}$ Cortical LGI growth was thought to be related to the increase in dendritic arborization, the growth of the terminal axonal arborization, synaptogenesis, and gial proliferation.27/28 Thus, SBM analyses provide an important way to investigate the cortical alterations induced by ECT in MDD patients. Until now, only three studies have been performed and only identified changes in $\mathrm{CT}$ in MDD patients after ECT $10 \mid 29[30$ However, the cortical alterations induced by ECT still needs to be fully elucidated. Given the specificity of CT, SA and LGI, investigations of cortical alterations using multidimensional structural patterns might provide novel views to understand the mechanism underlying the therapeutic efficacy and side effects of ECT in MDD patients.

In this study, we investigated the cortical alterations induced by ECT in $23 \mathrm{MDD}$ patients by examining the vertex-wise and regional $\mathrm{CT}, \mathrm{SA}$ and LGI using the SBM approach. Paired two-sample $t$-tests were performed to explore the variations in CT, SA and LGI across the whole brain cortex as well as across 68 anatomically defined cortical regions in MDD patients before and after ECT. Partial correlational analyses were performed to explore the relationship between the changes in $\mathrm{CT} / \mathrm{SA} / \mathrm{LGI}$ in ECT-related regions during the whole brain analyses as well as regional analyses and the changes of the clinical scores in the MDD patients with age, gender and education as covariates. Moreover, multivariate pattern analysis using linear support vector machine (SVM) was applied to investigate whether the changed morphological measurements can be an effective index to classify MDD patients before and after ECT.

\section{Materials and Methods}

\subsection{Participants}

Patients were recruited from the Anhui Mental Health Center between 2012 and 2015, were diagnosed with MDD according to the Diagnostic and Statistical Manual of Mental Disorders-IV criteria ${ }^{31}$ and were referred for ECT if they showed resistance to drug therapy or severe suicidal tendencies. In this study, we excluded patients who were left-handed, substance dependent, pregnant, had life-threatening somatic disease, neurological disorders, other comorbid mental disorders or MRI-related contraindications before ECT, resulting in 23 patients. All these patients continued to take anti-depressant drugs during the ECT administration. All patients provided written informed consent. The study was performed in accordance with the latest revision of the Declaration of Helsinki and was fully approved by the Anhui Medical University Ethics Committee. The demographics and clinical data of the MDD patients are listed in Table1. 
Table 1. Demographic characteristics of the participants.

\begin{tabular}{|c|c|c|c|}
\hline \multirow{2}{*}{$\frac{\text { Participants }}{\text { Number of subjects }}$} & \multicolumn{3}{|c|}{ MDD } \\
\hline & & 23 & \\
\hline Age & & $38.74 \pm 11.02($ years $)$ & \\
\hline Gender (male:female) & & $11: 12$ & \\
\hline Education & & $8.83 \pm 3.89($ years $)$ & \\
\hline Durations of illness & & $70.35 \pm 83.27$ (months) & \\
\hline Number of Treatment & & $7.26 \pm 2$ & \\
\hline On-mediation & & 23 patients & \\
\hline SSRIs & & 7 patients & \\
\hline SNRIs & & 4 patients & \\
\hline SSRIs+ SNRIs & & 1 patients & \\
\hline SSRIs+ NaSSAs & & 1 patients & \\
\hline SSRIs+ SARIs & & 1 patients & \\
\hline SSRIs+ Antipsychotics & & 9 patients & \\
\hline & Before ECT & After ECT & $p$ value \\
\hline AVLT-IR & $19.65 \pm 8.57$ & $17.13 \pm 6.47$ & $p=0.178$ \\
\hline AVLT-DR & $6.82 \pm 3.16$ & $3.91 \pm 3.78$ & $p<0.001$ \\
\hline HRSD scores & $22.22 \pm 4.74$ & $3.83 \pm 2.15$ & $p<0.001$ \\
\hline
\end{tabular}

Note: MDD, major depressive disorder; ECT, electroconvulsive therapy; SSRIs: selective serotonin reuptake inhibitors; SNRIs: serotonin-norepinephrine reuptake inhibitors; NASSAs: norepinephrine and specificity serotonergic antidepressants; SARIs: serotonin antagonist/reuptake inhibitors; AVLT-IR, immediate recall of Auditory Verbal Learning Test; AVLT-DR, delayed recall of Auditory Verbal Learning Test; HRSD, Hamilton Rating Scale for Depression. Paired two-sample $t$-tests were performed to test the difference of AVLT_IR, AVLT-DR and HRSD scores in MDD patients before and after ECT.

\subsection{Clinical measurements}

The severity of depression was assessed with the 17-item Hamilton Rating Scale for Depression $(\mathrm{HRSD}) \sqrt{32} 12-24 \mathrm{~h}$ before the first ECT session and 24-72 $\mathrm{h}$ after the last ECT session. Moreover, the Auditory Verbal Learning Test (AVLT) was also used to assess verbal episodic memory, which includes immediate recall AVLT (AVLT-IR) and delayed recall AVLT (AVLT-DR). Considering the difficulty of the classic AVLT, a simplified version was used in this study. At first, a list of 15 words was read to the subjects with the speed of one word per second. After presentation, the subjects were immediately asked to recall as many words as possible. The AVLT-IR was repeated three times and recalled words were recorded for three trials. After $10 \mathrm{~min}$, under the condition of no presentation before, the subjects were instructed to recall the 15 words presented during AVLT-IR and AVLT-DR. The total scores for AVLT-IR (trial 1-3) and AVLT-DR were separately analyzed.

\subsection{ECT procedures}

All patients underwent modified bi-frontal ECT using a Thymatron System IV Integrated ECT Instrument (Somatics, Lake Bluff, IL, USA). In our study, the first three ECT sessions were administered on consecutive days, and the subsequent ECT sessions were conducted every other day with a break over the weekends until patients reached symptom remission. The criterion for remission was defined as the HRSD score of patient was not more than 7 . The mean of the total duration of ECT sessions was $14.6 \pm 5.8$ days. And the mean number of treatment was $7.26 \pm 2$. The initial stimulation strength was set according to the age of each participant, and evenly increased until seizures were visually observed. All patients were given propofol anesthesia during the ECT. To help the patients relax their musculature and suppress the secretion of glands, succinylcholine and atropine were additionally administered. Moreover, we also used electroencephalography to monitor the seizure activity! 15$] 33$ 


\subsection{MRI data acquisition}

All patients underwent two scans $(12-24 \mathrm{~h}$ before the first ECT session and 24-72 h after the last ECT session) at the First Affiliated Hospital of Anhui Medical University using a clinical 3.0 T MRI scanner (Signa HDxt 3.0 T, GE Healthcare, Buckinghamshire, UK). The subjects were instructed to keep their eyes closed, stay relaxed and awake, and not to think of anything in particular during the scan. The parameters of the T1-weighted anatomic images were as follows: repetition time $=8.676 \mathrm{~ms}$, echo time $=3.184 \mathrm{~ms}$, inversion time $=800 \mathrm{~ms}$, flip angle $=8^{\circ}$, field of view $=256 \times 256 \mathrm{~mm}^{2}$, matrix size $=256 \times 256$, slice thickness $=1 \mathrm{~mm}$, voxel size $=1 \times 1 \times 1 \mathrm{~mm}^{3}$ and sections $=188$.

\subsection{Cortical reconstruction and longitudinal analysis}

To reduce within subject variability, longitudinal analysis were performed using the longitudinal processing pipeline ${ }^{\sqrt{36}}$ in FreeSurfer 5.0. (http://surfer.nmr.mgh.harvard.edu/). The longitudinal design can significantly reduce the confounding effect of inter-individual morphological variability by using each subject as his or her own control, and be unbiased with respect to any time point. It mainly consisted of three steps: (1) CROSS: all time points of all subjects were processed independently using the fully automated FreeSurfer "recon-all" standard procedure to obtain the information for constructing the subject template in the next step; (2) BASE: for each subject, a template was created from all time points to estimate average subject anatomy. An unbiased median image is used as the template and a full segmentation and surface reconstruction was performed; and (3) LONG: every time point is processed "longitudinally". The longitudinal processing of each time point was initialized with information from the subject template and the results of step BASE to reduce variability.

This process consisted of the following procedures: (1) spatial normalization and nonuniform intensity: all inputs are resampled to the unbiased template voxel space to further reduce variability. A nonparametric nonuniform intensity normalization was used to correct the acquisition bias fields in this procedure; (2) Talairach registration: transferring the map to Talairach coordinate system using a single global affine transformation; (3) brainmask creation: the brain mask is constructed as the union of the registered brain masks across time and is kept constant for all time points to reduce variability; (4) normalization and atlas registration; (5) subcortical segmentation: a fused segmentation is created for each time point by an intensity based probabilistic voting scheme; (6) surfaces reconstruction: similar to regular cortical surface construction in Freesurfer, but the white and pial surfaces in each time point are initialized with the surfaces from the template to demonstrate an implicit vertex correspondence; and (7) cortical atlas registration and parcellations: establishing a coordinate system for the cortex by warping individual surface models into register with a spherical atlas in a way that aligns the cortical folding patterns. For more detail information, please refer to papers by Reuter et al [36] 38 and website (https://surfer.nmr.mgh.harvard.edu/ fswiki/LongitudinalProcessing).

The CT was calculated as the closest distance from the gray/white boundary to the gray/ cerebrospinal fluid boundary at each vertex on the tessellated surface using T-average algorithm ${ }^{39}$ The SA for each vertex was calculated by assigning onethird of the area of each triangle to each of its vertices on the pial surface, representing the area of the tessellated triangles linked to the vertex. These two parameters were the output of FreeSurfer with its standard processing pipeline. The LGI, a measurement of cortical gyrification, was obtained with three main steps: (1) generating an artificial outer surface from the outer hull; (2) calculating the LGI of each vertex on the outer surface by measuring the ratio of local SA to the outer hull layer that tightly wraps the pial surface $\stackrel{40}{,}$ and (3) deriving the LGI map by redistributing the outer surface LGI values to each vertex on the pial surface. It is an indication of the sulcal cortex buried in its locality and thus denotes the extent of cortical folding [4]

\subsection{Vertex-wise $S B M$ analyses}

The CT, SA and LGI of the left and right hemisphere were smoothed with a circularly symmetric Gaussian kernel of $5 \mathrm{~mm}$ full width half maximum to improve signal to noise ratio and to further provide a normal distribution of the results. Between-group differences in CT, SA and LGI were investigated within the Qdec (query, design, estimate, contrast) application 
of FreeSurfer (https://surfer.nmr.mgh.harvard. edu/fswiki/Qdec) with paired two-sample $t$ test model in MDD patients before and after ECT. Finally, the results were corrected for multiple comparisons using Monte Carlo simulation (a precached cluster-wise level of $p<0.05$, a voxel-wise level of $p<0.001$, and sign: absolute).

\subsection{Regional $C T, S A$ and LGI analyses}

To increase the signal-to-noise by searching functionally homologous regions, CT, SA and LGI were averaged within each of the 68 anatomically defined regions from the Desikan-Killiany Atlas (Table 2) during the FreeSurfer segmentation procedures. Paired two-sample $t$-tests were performed to explore the regional differences in CT, SA and LGI in MDD patients before and after ECT using SPSS. A strict Bonferroni correction with $p<0.05 / 68$ was used as the threshold value of significance for the multiple comparisons.

\subsection{Correlational analyses}

Partial correlational analyses were performed to explore the relationship between the changes in $\mathrm{CT}$, SA and LGI in ECT-related regions during the whole brain analysis as well as in the anatomically defined cortical regions and the changes in AVLT-IR, AVLTDR and HRSD scores in the MDD patients with age, gender and education as covariates. All correlational analyses were performed using SPSS 19. The significant level was set at $p<0.05$, Bonferroni correction. To show the trend between changed morphological measurements and changed behavioral performances, all the correlations with $p<0.05$ were also shown.

\subsection{Multivariate pattern analysis using $S V M$}

To explore whether the identified neural indices can effectively classify MDD patients before and after ECT, a linear SVM approach within a library for SVMs (LIBSVMs) toolkit running on MATLAB (https://www.csie.ntu.edu.tw/ cjlin/libsvm/) was performed. The mean vertex-wise $\mathrm{CT}, \mathrm{SA}$ and LGI, as well as the mean regional $\mathrm{CT}$ and $\mathrm{SA}$ in all ECT-related regions were used as the features
Table 2. Cortical regions and abbreviations included in the Desikan-Killiany atlas performed with FreeSurferv5.1.0.

\begin{tabular}{ll}
\hline \multicolumn{1}{c}{ Region } & Abbreviations \\
\hline Banks of the superior temporal sulcus & bSTS \\
Caudal anterior cingulate & CAR \\
Caudal middle frontal gyrus & cMFG \\
Cuneus & CUN \\
Entorhinal cortex & EC \\
Fusiform gyrus & FG \\
Inferior parietal gyrus & IPG \\
Inferior temporal gyrus & ITG \\
Isthmus cingulate & IC \\
Lateral occipital gyrus & LOG \\
Lateral orbitofrontal gyrus & LFGor \\
Lingual gyrus & LG \\
Medial orbitofrontal gyrus & MFGor \\
Middle temporal gyrus & MTG \\
Parahippocampal gyrus & ParaHIPP \\
Paracentral gyrus & ParaCG \\
Pars opercularis & pOPER \\
Pars orbitalis & pORB \\
Pars triangularis & pTRI \\
Pericalcarine cortex & PeriCAL \\
Postcentral gyrus & PostCG \\
Posterior cingulate & PCC \\
Precentral gyrus & PreCG \\
Precuneus & PreCUN \\
Rostral anterior cingulate & RAC \\
Rostral middle frontal gyrus & rMFG \\
Superior frontal gyrus & SFG \\
Superior parietal gyrus & SPG \\
Superior temporal gyrus & STG \\
Supramarginal gyrus & SMG \\
Frontal pole & FP \\
Temporal pole & TP \\
Transverse temporal gyrus & \\
Insula & \\
\hline & \\
\hline
\end{tabular}

for discrimination (38 features). ReliefF ${ }^{42}$ was used to obtain the optimal combination of these features. A leave-one-out cross-validation strategy was used because of our limited number of samples to estimate the generalization ability of our classifier. The receiver operating characteristic (ROC) curve and area under curve (AUC) were used to delineate the classification ability.

\section{Results}

\subsection{Clinical measurements}

The paired $t$-tests identified significantly decreased HRSD scores in MDD patients after ECT $(p<$ 
0.001), indicating excellent therapeutic effects of ECT. There was no significant difference between the AVLT-IR in MDD patients after ECT compared to that before $\operatorname{ECT}(p=0.178)$. There were significantly decreased AVLT DR scores in MDD patients after $\operatorname{ECT}(p<0.001)$.

\subsection{Effects of ECT on $C T$}

Increased vertex-wise $\mathrm{CT}$ was observed in the left temporal pole (TP), left fusiform gyrus (FG), left inferior parietal gyrus (IPG), left insula (INS) and visual part of the left FG, as well as in the right lateral occipital gyrus (LOG), right supramarginal gyrus (SMG), right superior temporal gyrus (STG), right INS and right precentral gyrus (PreCG) in MDD patients after ECT (Fig. 1(a)). Meanwhile, increased regional $\mathrm{CT}$ was observed in the bilateral FG, bilateral parahippocampus (ParaHIPP) and bilateral inferior temporal gyrus (ITG), as well as in the left STG, left IPG, left INS, left banks of the superior temporal sulcus (bSTS), right superior frontal gyrus (SFG) and right rostral anterior cingulate (RAC) in the MDD patients after ECT (Fig. (1) and Table 3). Moreover, the increased vertex-wise and regional $\mathrm{CT}$ was also found in the left (TP), (FG) and bilateral INS in MDD patients after ECT (Fig. 1(c)).

\subsection{Effects of ECT on $S A$}

Increased vertex-wise SA was found in the left FG, left SMG, left INS and left lingual gyrus (LG), as well as in the right IPG, right STG, right ITG and right postcentral gyrus (PostCG) (Fig. 2(a)). Additionally, significantly increased regional SA of the bilateral FG, left rostral middle frontal gyrus (rMFG), left INS and right caudal middle frontal gyrus (cMFG) was also found in MDD patients after ECT (Fig. 2(b)). Moreover, the increased vertex-wise and regional SA was mainly found in the left FG and INS in MDD patients after ECT (Fig. 2(c)). The mean regional SA of these regions in MDD patients before and after ECT is listed in Table 4.

\section{(a)}

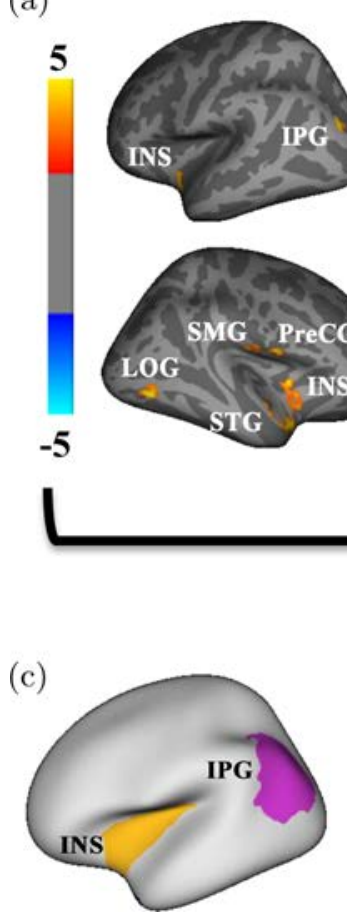

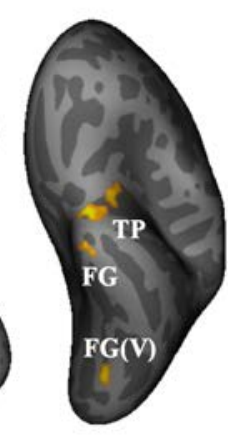

(b)
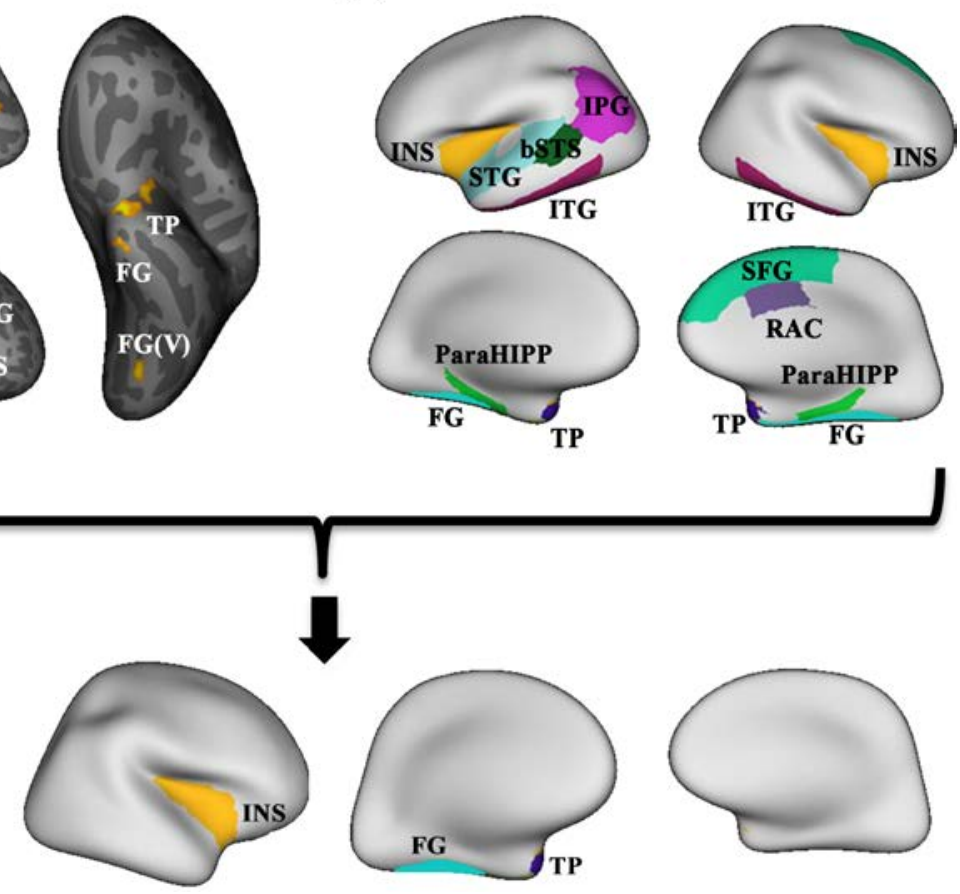

Fig. 1. Vertex-wise and regional analyses identified altered CT in MDD after ECT. All data were processed using the longitudinal pipelines in FreeSurfer. Paired $t$-tests were performed to identify the difference in vertex-wise CT in MDD before and after ECT. The significance level was set at a voxel-level of $p<0.001$ and a cluster-level of $p<0.05$, corrected by Monte Carlo simulations. Paired $t$-tests were performed to identify the differences in the regional CT in the MDD patients before and after ECT, and a strict Bonferroni correction with $p<0.05 / 68$ was used as the threshold of significance for the multiple comparisons. All abbreviations of the brain regions are listed in Table 2 
Table 3. Cortical regions that showed significantly increased regional CT in the MDD patients after ECT. Paired two-sample $t$-tests were performed to explore the regional differences in MDD patients before and after ECT using SPSS. A strict Bonferroni correction with $p<0.05 / 64$ was used as the threshold of significance for the multiple comparisons.

\begin{tabular}{lccccc}
\hline Regions & Before ECT & After ECT & $p$ value & Cohen's $d$ value & $t$ value \\
\hline bSTS.L & $2.290 \pm 0.178$ & $2.359 \pm 0.187$ & $4.9 \times 10^{-5}$ & 0.377 & -5.028 \\
FG.L & $2.736 \pm 0.155$ & $2.805 \pm 0.151$ & $1.3 \times 10^{-4}$ & 0.450 & -4.628 \\
IPG.L & $2.350 \pm 0.132$ & $2.408 \pm 0.114$ & $3.3 \times 10^{-5}$ & 0.470 & -5.191 \\
ParaHIPP.L & $2.629 \pm 0.315$ & $2.696 \pm 0.310$ & $1.6 \times 10^{-4}$ & 0.214 & -4.296 \\
STG.L & $2.512 \pm 0.140$ & $2.572 \pm 0.155$ & $2.6 \times 10^{-4}$ & 0.406 & -4.366 \\
TP.L & $3.686 \pm 0.260$ & $3.842 \pm 0.280$ & $7.0 \times 10^{-5}$ & 0.577 & -4.882 \\
INS.L & $2.925 \pm 0.128$ & $3.021 \pm 0.149$ & $9.6 \times 10^{-9}$ & 0.691 & -8.899 \\
FG.R & $2.756 \pm 0.149$ & $2.827 \pm 0.167$ & $3.1 \times 10^{-5}$ & 0.448 & -5.212 \\
ITG.R & $2.727 \pm 0.142$ & $2.802 \pm 0.154$ & $4.5 \times 10^{-5}$ & 0.506 & -5.026 \\
ParaHIPP.R & $2.652 \pm 0.271$ & $2.705 \pm 0.248$ & $2.9 \times 10^{-4}$ & 0.204 & -4.296 \\
RAC.R & $2.596 \pm 0.222$ & $2.685 \pm 0.238$ & $1.5 \times 10^{-4}$ & 0.269 & -4.548 \\
SFG.R & $2.613 \pm 0.145$ & $2.691 \pm 0.141$ & $1.9 \times 10^{-4}$ & 0.545 & -4.460 \\
TP.R & $3.875 \pm 0.273$ & $4.000 \pm 0.289$ & $1.1 \times 10^{-4}$ & 0.444 & -4.674 \\
INS.R & $2.900 \pm 0.119$ & $2.986 \pm 0.095$ & $7.4 \times 10^{-7}$ & 0.798 & -6.822 \\
\hline
\end{tabular}

(a)
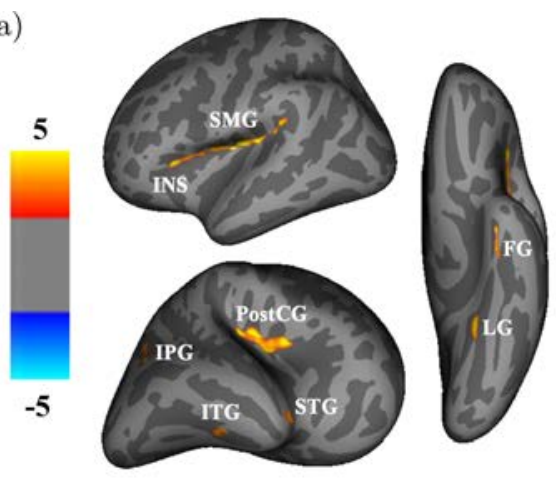

(b)
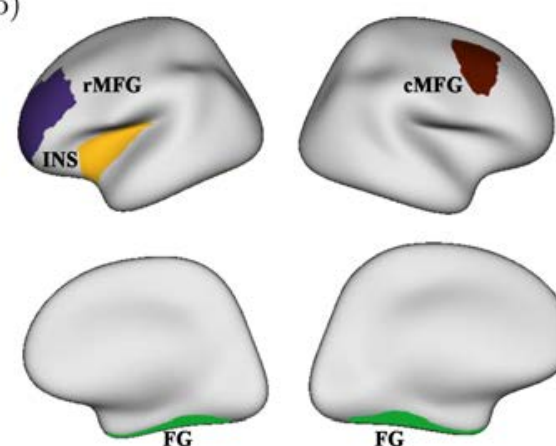

(c)
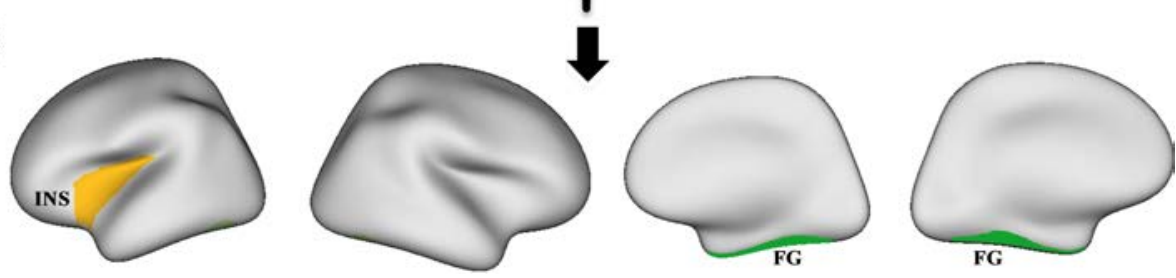

Fig. 2. Vertex-wise and regional analyses identified altered SA in MDD patients after ECT. All data were processed using the longitudinal pipelines in FreeSurfer. Paired $t$-tests were performed to identify the difference in vertex-wise SA in MDD patients before and after ECT. The significance level was set at a voxel-level of $p<0.001$ and a cluster-level of $p<0.05$, corrected by Monte Carlo simulations. Paired $t$-tests were performed to identify the difference in the regional SA in MDD patients before and after ECT, and a strict Bonferroni correction with $p<0.05 / 68$ was used as the threshold of significance for the multiple comparisons. All abbreviations of the brain regions are listed in Table 2 
Table 4. Cortical regions that showed significantly increased regional SA in the MDD patients after ECT. Paired two-sample $t$-tests were performed to explore the regional differences in MDD patients before and after ECT using SPSS. A strict Bonferroni correction with $p<0.05 / 64$ was used as the threshold of significance for the multiple comparisons.

\begin{tabular}{lccccc}
\hline \multicolumn{1}{c}{ Regions } & Before ECT & After ECT & $p$ value & Cohen's $d$ value & $t$ value \\
\hline FG.L & $3433.91 \pm 365.60$ & $3484.00 \pm 382.57$ & $7.2 \times 10^{-5}$ & 0.133 & -4.87 \\
rMFG.L & $5698.26 \pm 870.20$ & $5762.34 \pm 906.25$ & $9.2 \times 10^{-5}$ & 0.072 & -4.76 \\
INS.L & $2290.69 \pm 299.93$ & $2340.34 \pm 311.63$ & $3.6 \times 10^{-6}$ & 0.162 & -6.13 \\
cMFG.R & $1982.26 \pm 321.90$ & $2004.21 \pm 325.24$ & $9.7 \times 10^{-5}$ & 0.067 & -4.74 \\
FG.R & $3246.86 \pm 405.22$ & $3295.56 \pm 402.21$ & $6.4 \times 10^{-5}$ & 0.120 & -4.91 \\
\hline
\end{tabular}

\subsection{Effects of ECT on LGI}

We only found that the vertex-wise LGI of the left middle temporal gyrus (MTG) significantly increased in the MDD patients after ECT (Fig. 3).

\subsection{Correlational analyses}

After multiple comparisons, only the changes in the regional $\mathrm{CT}$ in the left IPG were significantly correlated with the changes in the ALVT_DR score in MDD patients after ECT (Fig. (4).

In addition, we also found marginal correlations which showed the trend of the changed cortical measurements and behavioral performance (Fig. 5), such

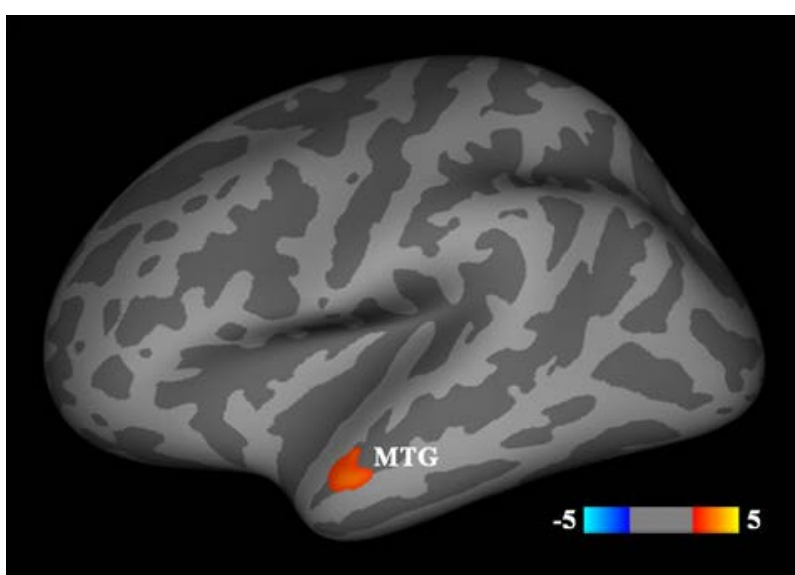

Fig. 3. Vertex-wise LGI of the MTG was significantly increased in the MDD patients after ECT. All data were processed using the longitudinal pipelines in FreeSurfer. Paired $t$-tests were performed to identify the difference in vertex-wise LGI in MDD patients before and after ECT. The significance level was set at a voxel-level of $p<0.001$ and a cluster-level of $p<0.05$, corrected by Monte Carlo simulations. All other abbreviations of the brain regions are listed in Table 2

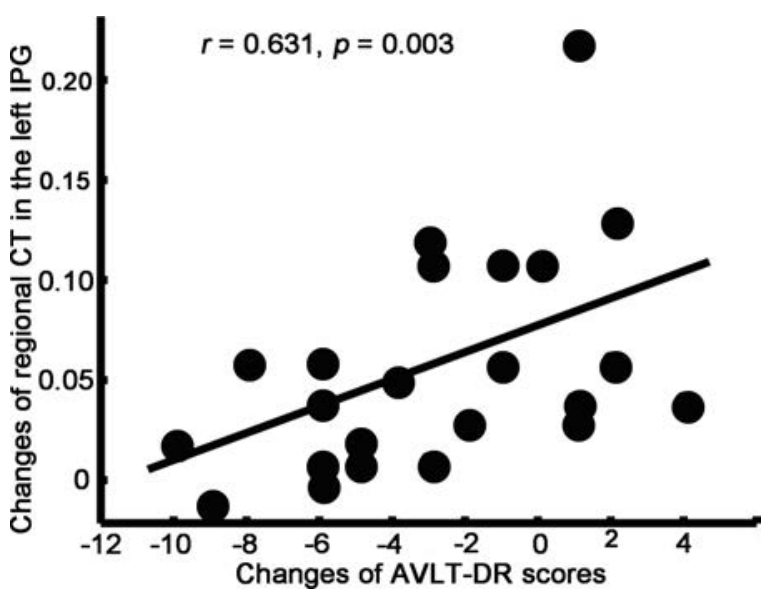

Fig. 4. Partial correlations were performed with age, gender and education as covariates. After multiple comparisons, changes in the regional CT in the left IPG were significantly correlated with the changes in the ALVT_DR score in MDD patients after ECT.

as the changes in vertex-wise $\mathrm{CT}$ in the right LOG were marginally correlated with the changes in the HRSD scores, the changes in vertex-wise CT in the right PreCG were marginally correlated with the changes in the ALVT_DR scores, the changes in vertex-wise SA in the left INS were marginally correlated with the changes in the AVLT_IR scores, the changes in regional SA in the left INS were marginally correlated with the changes in the HRSD scores, and the changes in vertex-wise SA in the right ITG were marginally correlated with the changes in the AVLT_DR scores in MDD after ECT.

\section{6. $\quad S V M$ results}

With a combination of eight ECT-related features, including the vertex wise CT in left IPG, left TP, 


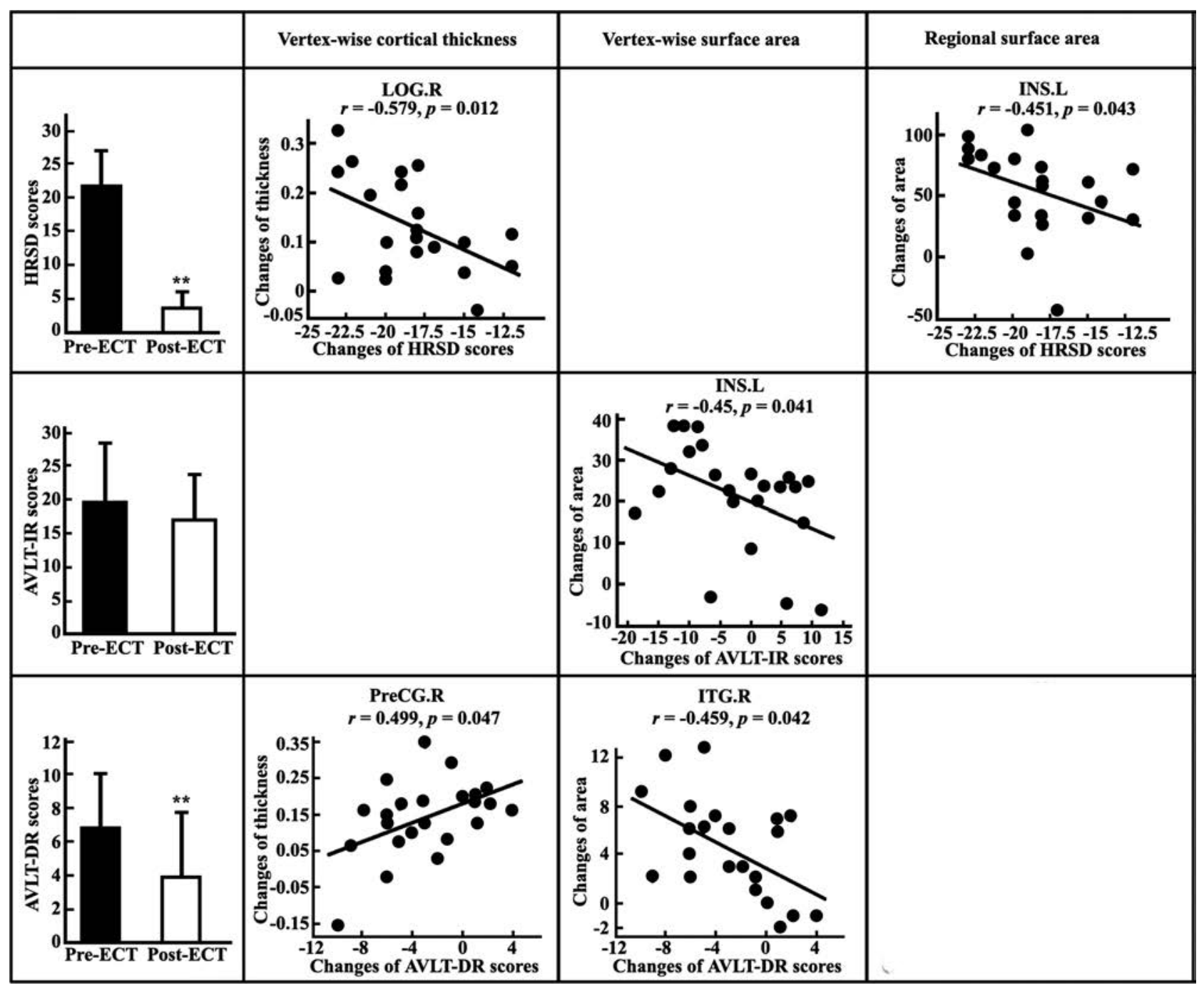

Fig. 5. Correlations showed the trend of the changed morphological matrices and behavioral performances in MDD patients. Partial correlations were performed with age, gender and education as covariates.

right INS and right LOC and regional CT in the left FG, left TP, right FG and right ITG, SVM achieved a good performance with $\mathrm{AUC}=0.77$ to classify the MDD patients after ECT from MDD patients (Fig. 6).

\section{Discussion}

In the current study, we described that ECT induced cortical changes in widespread cortical regions, especially in the left INS and left FG in the MDD patients. Furthermore, correlations were also identified between the changes in the CT/SA in ECTrelated regions and the changes in the clinical measurements in the MDD patients.
The longitudinal cortical alterations induced by ECT were widely observed in temporal and frontal regions, providing further evidence for the neuroplastic effects of ECT identified in animal and human experiments. ${ }^{15}$ Previous investigations based on animal models showed that repetitive electroconvulsive shock elicited widespread cell proliferation, $\frac{43}{\text { par- }}$ ticularly in the hippocampus $\stackrel{444 / 45}{\text { amygdald } 3}$ and prefrontal cortex ${ }^{[6 / 47}$ In addition, many structural studies reported increase in the gray matter volume in the hippocampus and amygdala, $15[48,51$ which were accompanied by associations with symptom improvement in MDD patients after ECT. However, the number of investigations of ECT-related cortical alterations in other cortical areas is relatively small. 


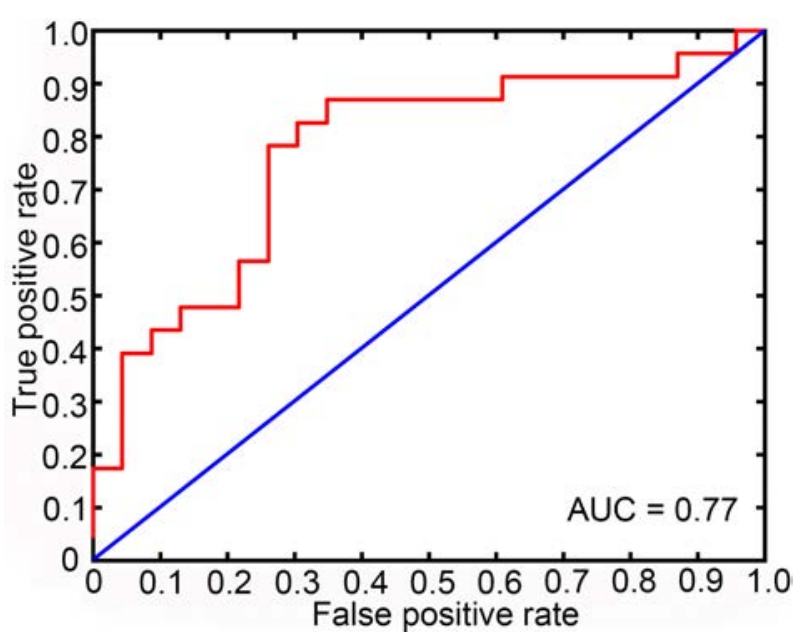

Fig. 6. The ROC curve of SVM classifier. A linear SVM approach was performed. The mean vertex-wise CT, SA and LGI, as well as the mean regional $\mathrm{CT}$ and $\mathrm{SA}$ in all ECT-related regions were used as the features for discrimination (38 features). After using ReliefF, a combination of eight features was served as the best solution for the SVM classifier.

To our knowledge, only three studies have been performed to reveal the alterations in $\mathrm{CT}$ related to ECT in MDD patients 1022930 The first study by Sartorius et al ${ }^{10}$ showed increasing $\mathrm{CT}$ in the TP and INS in MDD patients after a mean of $11.3 \pm 4.8$ ECT treatments using voxel-based cortical thickness, and further corroborating with VBM results. However, no significant correlations with changes in psychopathology were found in his study. Then, two studies were performed using SBM at the same year, one revealed ${ }^{29}$ significant increases in $\mathrm{CT}$, including the bilateral TP, ITG, MTG and INS in MDD patients after a mean of $18 \pm 7$ ECT treatments; the other ${ }^{30}$ showed increased CT after a mean of 11.2 ECT treatments in the bilateral ACC, ITG, STG, ParaHIPP, FG and prefrontal areas in MDD patients. In our study, patients received a mean of $7.26 \pm 2$ ECT treatments and showed cortical alterations in widespread cortical regions. Our findings were supported by those of the previous studies, and we further extended their findings by showing a widespread increase in CT, SA and LGI, as well as significant correlations with changes of clinical measurement in the MDD patients, which mainly reflect distinct alterations in the developmental trajectories of cortical structures related to ECT.
We found that both the vertex-wise and regional CT and SA significantly increased in the left INS and showed correlations with the clinical measurements. Our current findings were supported by those of previous studies in MDD patients. Using VBM analysis, increased grey matter volume in the INS was found in the patients with late-life depression after ECT ${ }^{14}$ Dukart and colleagues reported that right unilateral ECT was correlated with regional increases in local grey matter volume of the INS 17 Using longitudinal analysis, van Eijndhoven et al.29] also revealed significantly increased $\mathrm{CT}$ in the bilateral INS, as well as an association between changes in the mean $\mathrm{CT}$ of the right INS and the changes in the HRSD scores, a result that was consistent with our current findings. Moreover, the INS has also been reported to be related to treatment responses in MDD patients. ${ }^{[52]}$ An increasing amount of evidence, such as decreased interoceptive activity, altered connectivity, $\underset{53 \mid 54}{5}$ and grey matter reductions, $\frac{55}{,}$ has suggested that the INS is an important structure in the pathophysiology of MDD $\stackrel{55 / 56}{5}$ In addition, our findings suggested that ECT-induced cortical plasticity (CT and SA) of the left INS, and altered cortical properties were associated with treatment response, which suggested that the CT and SA of the left INS may serve as a potential neuroimaging biomarker for the therapeutic efficacy of ECT in MDD patients. Additionally, we found that the changes in $\mathrm{CT} / \mathrm{SA}$ in the left INS were negatively correlated with the changes in the AVLT_IR scores, showing the possibility that the increased CT/SA of the left INS may be associated with the memory deficits in MDD patients after ECT, indicating that normal functioning of the brain is a dynamic balance of processes.

We also found that both the vertex-wise and regional $\mathrm{CT}$ and $\mathrm{SA}$ were significantly increased in the left FG in the MDD patients after ECT. The FG is a key region in the ventral processing stream of visual information and complex feature detection, which seems to be specifically correlated with facial expression. $57-59$ Functional and structural abnormalities in the FG have been widely reported, with studies particularly reporting reduced FG activation during face processing in MDD patients. 60 Importantly, the fMRI signal in the left FG during affective face processing was found to be significantly associated with greater individual-level estimates of perceptual processing efficiency $[62$ A recent 
study revealed altered functional and effective connectivities between the FG and amygdala in MDD patients after ECT 15 With all this evidence, the increased $\mathrm{CT}$ and $\mathrm{SA}$ in the left FG in our current study indicated that ECT could improve facial emotion processing in MDD patients.

In the whole brain analysis of the LGI, significant hypergyrification in the left anterior MTG which is part of the default mode network ${ }^{63}$ was identified in the MDD patients after ECT. According to the tension-based theory of cortical morphogenesis, $[4]$ the LGI results from forces driving the extensive wiring of cortico-cortical connections along the brain surface, reflecting the early neurodevelopment of cortical connectivity. Under this theory, the hypergyrification in the left MTG might result from the increased connectivity due to the white matter alterations induced by the ECT in MDD patients. However, another mechanical model of brain convolutional development, which suggested that differential growth rates of cortical layers directly affect the degree of cortical folding, has also been used to explain the cortical folding ${ }^{65}$ In fact, increased rates of neurogenesis, synaptogenesis and glial proliferation were found in animal models after ECT $[3][6$ Therefore, the increased LGI in the left MTG might be associated with the reconfiguring of the cortical architectures in this region. Moreover, the MTG has been reported to have a reduced gray matter volume, lower amplitude of low-frequency fluctuations and altered functional connectivity in MDD patients. 66 Similar to our results, the regional homogeneity in the left MTG was reported to be increased after eight course of ECT in MDD patients $\frac{13}{13}$ These results collectively suggested that the structure and function of the left MTG might be associated with the mechanism of ECT in MDD. Although previous studies suggested that the LGI plays an important role in MDD patients, 69 this was the first study using LGI to directly demonstrate the cortical alterations induced by ECT in MDD patients. Our results suggested that the LGI is a useful index for estimating the cortical alterations, which providing new insight regarding the understanding of the mechanism underlying ECT in MDD patients.

Furthermore, the correlational analyses performed in the present study suggested close associations between the changes in the $\mathrm{CT}$ in right
LOG and the changes in the HRSD scores in MDD patients. The right LOG is part of the visual recognition circuit, which primarily participates in object identification ${ }^{73}$ and affect identification! ${ }^{74.75}$ Moreover, disrupted connectivity between the visual recognition circuit and the subgenual anterior cingulate cortex and significant associations with the HRSD scores were identified in MDD patients. .54 Our findings of a significant correlation between the changes in the $\mathrm{CT}$ in the right $\mathrm{LOG}$ and the changes in the HRSD scores in MDD patients indicated that ECT may alleviate symptoms by modulating the structure of the visual cognition network to reduce the negative bias in emotion recognition.

In addition, we also found correlations between the changes in the AVLT_ DR scores and the changes in the SA in the right ITG, as well as with the changes in the CT in the left IPL in the MDD patients. Although the left IPL is widely considered to play a pivotal role in language processing, ${ }^{76}$ a recent parcellation study revealed that the posterior subregions of the IPL were engaged in episodic memory and working memory using functional connectivity and behavioral domain analyses. $\frac{80}{10}$ The ITG has also been demonstrated to play an important role in working memory in several previous studies $\sqrt[81,82]{ }$ Moreover, depressed patients have been shown abnormal activations in the ITG when they intended to forget negative words, which was used as a useful feature to discriminate depressed participants from the control participants. ${ }^{83}$ Therefore, the correlations found in our study suggested that the cortical variations in the right ITG and left IPL in the memory network were closely associated with the memory impairment in MDD patients after ECT.

Although this paper provided new insight into the understanding of the mechanism underlying the therapeutic efficacy and side effects of ECT in MDD patients, some limitations still need to be addressed. First, our sample size was relatively small, making the statistical power weak especially the results of correlation analysis. Thus, any structural changes found in our study should be interpreted with caution, even though we performed strict multiple corrections. Moreover, these structural changes might also due to brain oedema since ECT can produce damage to the blood-brain barrier resulting in the brain oedema. $84 \mid 85$ Even though we didn't 
find any obvious brain oedema in T1 images of MDD patients after ECT, it is difficult to exclude its effects as there might be some oedema invisibly by MRI T1. Second, all the MDD patients were taking anti-depressant medications during ECT administrations even they showed resistance to them. Future studies of medications-naive patients are warranted to exclude the effects of anti-depressant medications. Third, we did not include normal controls because of the constraint of the longitudinal analysis method. However, we found that the altered surface measurements were closely correlated with altered clinical characteristics, suggesting that longitudinal analysis alone was enough to elucidate the mechanism of ECT. Finally, we only described structural alterations induced by ECT in the MDD patients, since structural alterations always associated with functional changes, fMRI and EEG ${ }^{86}$ are suited for investigating functional changing patterns induced by ECT in the MDD patients.

\section{Conclusions}

In conclusion, we described that the ECT induced significant increase in the CT, SA and LGI in a widespread of cortical regions, particularly in left INS and left FG in MDD patients reflecting distinct alterations in the developmental trajectories of cortical structures related to ECT. Further correlations were also identified between the changes in the $\mathrm{CT} / \mathrm{SA}$ in ECT-related regions and the changes in the clinical measurements in MDD patients. In addition, these changed morphological measurements were an effective index to classify the MDD patients after ECT from MDD patients. Taken together, our findings suggested that ECT may enhance cortical neuroplasticity to facilitate neurogenesis to remit depressive symptoms, and the cortical morphology is a good indicator for therapeutic efficacy of ECT.

\section{Acknowledgments}

This work was supported by Natural Science Foundation of Guangdong Province (No. 2017A030313744 and No. 2016A030313180), the Science and Technology innovation program of Shenzhen (No. JCYJ20160531184531506), the National Natural Science Foundation of China (Nos. 31500867, 61671440 and 61672510) and the Shenzhen Key Basic Research Grant (No. JCYJ20160331191401141).

\section{References}

1. U. E. R. Group, Efficacy and safety of electroconvulsive therapy in depressive disorders: A systematic review and meta-analysis, Lancet 361(9360) (2003) 799-808.

2. S. H. Lisanby, J. H. Maddox, J. Prudic, D. P. Devanand and H. A. Sackeim, The effects of electroconvulsive therapy on memory of autobiographical and public events, Arch. Gen. Psychiatry 57(6) (2000) 581-590.

3. M. Wennstrom, J. Hellsten and A. Tingstrom, Electroconvulsive seizures induce proliferation of NG2expressing glial cells in adult rat amygdala, Biol. Psychiatry 55(5) (2004) 464-471.

4. J. Hellsten, M. Wennstrom, P. Mohapel, C. T. Ekdahl, J. Bengzon and A. Tingstrom, Electroconvulsive seizures increase hippocampal neurogenesis after chronic corticosterone treatment, Eur. J. Neurosci. 16(2) (2002) 283-290.

5. F. Chen, T. M. Madsen, G. Wegener and J. R. Nyengaard, Repeated electroconvulsive seizures increase the total number of synapses in adult male rat hippocampus, Eur. Neuropsychopharmacol. J. Eur. Coll. Neuropsychopharmacol. 19(5) (2009) 329-338.

6. A. Kondratyev, R. Ved and K. Gale, The effects of repeated minimal electroconvulsive shock exposure on levels of mRNA encoding fibroblast growth factor-2 and nerve growth factor in limbic regions, Neurosci. 114(2) (2002) 411-416.

7. L. Santarelli, M. Saxe, C. Gross, A. Surget, F. Battaglia, S. Dulawa, N. Weisstaub, J. Lee, R. Duman, O. Arancio, C. Belzung and R. Hen, Requirement of hippocampal neurogenesis for the behavioral effects of antidepressants, Science 301(5634) (2003) 805-809.

8. P. W. Frankland, S. Kohler and S. A. Josselyn, Hippocampal neurogenesis and forgetting, Trends Neurosci. 36(9) (2013) 497-503.

9. T. Nickl-Jockschat, N. Palomero Gallagher, V. Kumar, F. Hoffstaedter, E. Brugmann, U. Habel, S. B. Eickhoff and M. Grozinger, Are morphological changes necessary to mediate the therapeutic effects of electroconvulsive therapy? Eur. Archives Psychiatry Clin. Neurosci. 266(3) (2016) 261-267.

10. A. Sartorius, T. Demirakca, A. Bohringer, C. Clemm von Hohenberg, S. S. Aksay, J. M. Bumb, L. Kranaster and G. Ende, Electroconvulsive therapy increases temporal gray matter volume and cortical thickness, Eur. Neuropsychopharmacol. 26(3) (2016) 506-517.

11. P. Nordanskog, U. Dahlstrand, M. R. Larsson, E. M. Larsson, L. Knutsson and A. Johanson, Increase in hippocampal volume after electroconvulsive therapy in patients with depression: A volumetric magnetic resonance imaging study, J. Ect. 26(1) (2010) 62-67.

12. G. Ende, D. F. Braus, S. Walter, W. Weber-Fahr and F. A. Henn, The hippocampus in patients treated 
with electroconvulsive therapy: A proton magnetic resonance spectroscopic imaging study, Arch. Gen. Psychiatry 57(10) (2000) 937-943.

13. H. Qiu, X. Li, W. Zhao, L. Du, P. Huang, Y. Fu, T. Qiu, P. Xie, H. Meng and Q. Luo, Electroconvulsive therapy-induced brain structural and functional changes in major depressive disorders: A longitudinal study, Med. Sci. Monitor Int. Med. J. Exp. Clin. Res. 22 (2016) 4577-4586.

14. F. Bouckaert, F. L. De Winter, L. Emsell, A. Dols, D. Rhebergen, M. Wampers, S. Sunaert, M. Stek, P. Sienaert and M. Vandenbulcke, Grey matter volume increase following electroconvulsive therapy in patients with late life depression: A longitudinal MRI study, J. Psychiatry Neurosci. 41(2) (2016) 105-114.

15. J. Wang, Q. Wei, T. Bai, X. Zhou, H. Sun, B. Becker, Y. Tian, K. Wang and K. Kendrick, Electroconvulsive therapy selectively enhanced feedforward connectivity from fusiform face area to amygdala in major depressive disorder, Social Cognitive Affective Neurosci. 12(12) (2017) 1983-1992.

16. M. Ota, T. Noda, N. Sato, M. Okazaki, M. Ishikawa, K. Hattori, H. Hori, D. Sasayama, T. Teraishi, D. Sone and H. Kunugi, Effect of electroconvulsive therapy on gray matter volume in major depressive disorder, J. Affect. Disord. 186 (2015) 186-191.

17. J. Dukart, F. Regen, F. Kherif, M. Colla, M. Bajbouj, I. Heuser, R. S. Frackowiak and B. Draganski, Electroconvulsive therapy-induced brain plasticity determines therapeutic outcome in mood disorders, Proc. Natl. Acad. Sci. U. S. A 111(3) (2014) 1156-1161.

18. D. Peng, F. Shi, G. Li, D. Fralick, T. Shen, M. Qiu, J. Liu, K. Jiang, D. Shen and Y. Fang, Surface vulnerability of cerebral cortex to major depressive disorder, PLoS One 10(3) (2015) e0120704.

19. G. Li, J. Nie, L. Wang, F. Shi, W. Lin, J. H. Gilmore and D. Shen, Mapping region-specific longitudinal cortical surface expansion from birth to 2 years of age, Cereb Cortex 23(11) (2013) 2724-2733.

20. G. Li, L. Wang, F. Shi, A. E. Lyall, W. Lin, J. H. Gilmore and D. Shen, Mapping longitudinal development of local cortical gyrification in infants from birth to 2 years of age, J. Neurosci. 34(12) (2014) 4228-4238.

21. A. Pontious, T. Kowalczyk, C. Englund and R. F. Hevner, Role of intermediate progenitor cells in cerebral cortex development, Dev. Neurosci. 30(1-3) (2008) 24-32.

22. C. la Fougere, S. Grant, A. Kostikov, R. Schirrmacher, P. Gravel, H. M. Schipper, A. Reader, A. Evans and A. Thiel, Where in vivo imaging meets cytoarchitectonics: The relationship between cortical thickness and neuronal density measured with highresolution $[18 \mathrm{~F}]$ flumazenil-PET, Neuroimage 56(3) (2011) 951-960.
23. M. F. Casanova and C. R. Tillquist, Encephalization, emergent properties and psychiatry: A minicolumnar perspective, The Neurosci. 14(1) (2008) 101-118.

24. J. Hill, T. Inder, J. Neil, D. Dierker, J. Harwell and D. Van Essen, Similar patterns of cortical expansion during human development and evolution, Proc. Natl. Acad. Sci. U.S.A. 107(29) (2010) 1313513140.

25. P. S. Goldman-Rakic, Morphological consequences of prenatal injury to the primate brain, Prog. Brain Res. 53 (1980) 1-19.

26. D. C. Van Essen, A tension-based theory of morphogenesis and compact wiring in the central nervous system, Nature 385(6614) (1997) 313-318.

27. Z. Petanjek, M. Judas, G. Simic, M. R. Rasin, H. B. Uylings, P. Rakic and I. Kostovic, Extraordinary neoteny of synaptic spines in the human prefrontal cortex, Proc. Natl. Acad. Sci. U.S.A. 108(32) (2011) 13281-13286.

28. Z. Petanjek, M. Judas, I. Kostovic and H. B. Uylings, Lifespan alterations of basal dendritic trees of pyramidal neurons in the human prefrontal cortex: A layer-specific pattern, Cereb Cortex 18(4) (2008) 915-929.

29. P. van Eijndhoven, P. Mulders, L. Kwekkeboom, I. van Oostrom, M. van Beek, J. Janzing, A. Schene and I. Tendolkar, Bilateral ECT induces bilateral increases in regional cortical thickness, Transl. Psychiatry 6(8) (2016) e874.

30. T. Pirnia, S. H. Joshi, A. M. Leaver, M. Vasavada, S. Njau, R. P. Woods, R. Espinoza and K. L. Narr, Electroconvulsive therapy and structural neuroplasticity in neocortical, limbic and paralimbic cortex, Transl. Psychiatry 6(6) (2016) e832.

31. American Psychiatric Association and American Psychiatric Association, Task Force on DSM-IV., Diagnostic and Statistical Manual of Mental Disorders: DSM-IV. 4th ed. (American Psychiatric Association, Washington, DC, 1994) xxvii, p. 886.

32. M. Hamilton, A rating scale for depression, J. Neurol. Neurosur. Ps. 23(1) (1960) 56-62.

33. Q. Wei, Y. Tian, Y. Yu, F. Zhang, X. Hu, Y. Dong, Y. Chen, P. Hu, X. Hu and K. Wang, Modulation of interhemispheric functional coordination in electroconvulsive therapy for depression, Transl. Psych. 4(9) (2014) e453.

34. J. Wang, Q. Wei, X. Yuan, X. Jiang, J. Xu, X. Zhou, Y. Tian and K. Wang, Local functional connectivity density is closely associated with the response of electroconvulsive therapy in major depressive disorder, J. Affect. Disord. 225 (2017) 658-664.

35. J. Wang, Q. Wei, L. Wang, H. Zhang, T. Bai, L. Cheng, Y. Tian and K. Wang, Functional reorganization of intra- and internetwork connectivity in major depressive disorder after electroconvulsive therapy, Human Brain Map 39(3) (2018) 1403-1411. 
36. M. Reuter, N. J. Schmansky, H. D. Rosas and B. Fischl, Within-subject template estimation for unbiased longitudinal image analysis, Neuroimage 61(4) (2012) 1402-1418.

37. M. Reuter, H. D. Rosas and B. Fischl, Highly accurate inverse consistent registration: A robust approach, Neuroimage 53(4) (2010) 1181-1196.

38. M. Reuter and B. Fischl, Avoiding asymmetryinduced bias in longitudinal image processing, $\mathrm{Neu}$ roimage 57(1) (2011) 19-21.

39. B. Fischl and A. M. Dale, Measuring the thickness of the human cerebral cortex from magnetic resonance images, Proc. Natl. Acad. Sci. U.S.A. 97(20) (2000) 11050-11055.

40. M. Schaer, M. B. Cuadra, L. Tamarit, F. Lazeyras, S. Eliez and J. P. Thiran, A surface-based approach to quantify local cortical gyrification, IEEE Trans. Med. Imag. 27(2) (2008) 161-170.

41. P. A. Kelly, E. Viding, G. L. Wallace, M. Schaer, S. A. De Brito, B. Robustelli and E. J. McCrory, Cortical thickness, surface area and gyrification abnormalities in children exposed to maltreatment: Neural markers of vulnerability? Biol. Psychiatry 74(11) (2013) 845-852.

42. M. E. Stokes and S. Visweswaran, Application of a spatially-weighted Relief algorithm for ranking genetic predictors of disease, BioData Min. 5(1) (2012) 20.

43. T. M. Madsen, A. Treschow, J. Bengzon, T. G. Bolwig, O. Lindvall and A. Tingstrom, Increased neurogenesis in a model of electroconvulsive therapy, Biol. Psychiat 47(12) (2000) 1043-1049.

44. T. D. Perera, J. D. Coplan, S. H. Lisanby, C. M. Lipira, M. Arif, C. Carpio, G. Spitzer, L. Santarelli, B. Scharf, R. Hen, G. Rosoklija, H. A. Sackeim and A. J. Dwork, Antidepressant-induced neurogenesis in the hippocampus of adult nonhuman primates, $J$. Neurosci. 27(18) (2007) 4894-4901.

45. Y. Imoto, E. Segi-Nishida, H. Suzuki and K. Kobayashi, Rapid and stable changes in maturation-related phenotypes of the adult hippocampal neurons by electroconvulsive treatment, Mol. Brain 10(1) (2017) 8.

46. T. M. Madsen, D. D. Yeh, G. W. Valentine and R. S. Duman, Electroconvulsive seizure treatment increases cell proliferation in rat frontal cortex, $\mathrm{Neu}$ ropsychopharmacol. 30(1) (2005) 27-34.

47. D. Inta, J. M. Lima-Ojeda, T. Lau, W. Tang, C. Dormann, R. Sprengel, P. Schloss, A. Sartorius, A. Meyer-Lindenberg and P. Gass, Electroconvulsive therapy induces neurogenesis in frontal rat brain areas, Plos One 8(7) (2013) e69869.

48. C. C. Abbott, T. Jones, N. T. Lemke, P. Gallegos, S. M. McClintock, A. R. Mayer, J. Bustillo and V. D. Calhoun, Hippocampal structural and functional changes associated with electroconvulsive therapy response, Transl. Psychiatry 4 (2014) e483.
49. A. Jorgensen, P. Magnusson, L. G. Hanson, T. Kirkegaard, H. Benveniste, H. Lee, C. Svarer, J. D. Mikkelsen, A. Fink-Jensen, G. M. Knudsen, O. B. Paulson, T. G. Bolwig and M. B. Jorgensen, Regional brain volumes, diffusivity and metabolite changes after electroconvulsive therapy for severe depression, Acta Psychiatrica Scandinavica 133(2) (2016) 154-164.

50. I. Tendolkar, M. van Beek, I. van Oostrom, M. Mulder, J. Janzing, R. O. Voshaar and P. van Eijndhoven, Electroconvulsive therapy increases hippocampal and amygdala volume in therapy refractory depression: A longitudinal pilot study, Psychiatry Res. 214(3) (2013) 197-203.

51. S. H. Joshi, R. T. Espinoza, T. Pirnia, J. Shi, Y. Wang, B. Ayers, A. Leaver, R. P. Woods and K. L. Narr, Structural plasticity of the hippocampus and amygdala induced by electroconvulsive therapy in major depression, Biol. Psychiatry 79(4) (2016) 282-292.

52. D. Sliz and S. Hayley, Major depressive disorder and alterations in insular cortical activity: A review of current functional magnetic imaging research, Front. Human Neurosci. 6 (2012) 323.

53. J. A. Avery, W. C. Drevets, S. E. Moseman, J. Bodurka, J. C. Barcalow and W. K. Simmons, Major depressive disorder is associated with abnormal interoceptive activity and functional connectivity in the insula, Biol. Psychiat. 76(3) (2014) 258-266.

54. H. Wu, H. Sun, J. Xu, Y. Wu, C. Wang, J. Xiao, S. She, J. Huang, W. Zou, H. Peng, X. Lu, G. Huang, T. Jiang, Y. Ning and J. Wang, Changed hub and corresponding functional connectivity of subgenual anterior cingulate cortex in major depressive disorder, Front. Neuroanatomy 10 (2016) 120.

55. R. Sprengelmeyer, J. D. Steele, B. Mwangi, P. Kumar, D. Christmas, M. Milders and K. Matthews, The insular cortex and the neuroanatomy of major depression, J. Affect. Disord. 133(1-2) (2011) 120127.

56. M. Nagai, K. Kishi and S. Kato, Insular cortex and neuropsychiatric disorders: A review of recent literature, Eur. Psychiatry J. Assoc. Eur. Psychiatrists 22(6) (2007) 387-394.

57. N. Kanwisher, J. McDermott and M. M. Chun, The fusiform face area: A module in human extrastriate cortex specialized for face perception, J. Neurosci. 17(11) (1997) 4302-4311.

58. M. J. Farah and G. K. Aguirre, Imaging visual recognition: PET and fMRI studies of the functional anatomy of human visual recognition, Trends Cognitive Sci. 3(5) (1999) 179-186.

59. W. Zhang, J. Wang, L. Fan, Y. Zhang, P. T. Fox, S. B. Eickhoff, C. Yu and T. Jiang, Functional organization of the fusiform gyrus revealed with connectivity profiles, Human Brain Mapping 37(8) (2016) 3003-3016. 
60. E. Henje Blom, C. G. Connolly, T. C. Ho, K. Z. LeWinn, N. Mobayed, L. Han, M. P. Paulus, J. Wu, A. N. Simmons and T. T. Yang, Altered insular activation and increased insular functional connectivity during sad and happy face processing in adolescent major depressive disorder, J. Affect. Disord. 178 (2015) 215-23.

61. T. C. Ho, G. Yang, J. Wu, P. Cassey, S. D. Brown, N. Hoang, M. Chan, C. G. Connolly, E. Henje-Blom, L. G. Duncan, M. A. Chesney, M. P. Paulus, J. E. Max, R. Patel, A. N. Simmons and T. T. Yang, Functional connectivity of negative emotional processing in adolescent depression, J. Affect. Disord. 155 (2014) 65-74.

62. T. C. Ho, S. Zhang, M. D. Sacchet, H. Weng, C. G. Connolly, E. Henje Blom, L. K. Han, N. O. Mobayed and T. T. Yang, Fusiform gyrus dysfunction is associated with perceptual processing efficiency to emotional faces in adolescent depression: A model-based approach, Front. Psychol. 7 (2016) 40.

63. J. Xu, J. Wang, L. Fan, H. Li, W. Zhang, Q. Hu and T. Jiang, Tractography-based parcellation of the human middle temporal gyrus, Sci. Rep. 5 (2015) 18883.

64. D. C. VanEssen, A tension-based theory of morphogenesis and compact wiring in the central nervous system, Nature 385(6614) (1997) 313-318.

65. D. P. Richman, R. M. Stewart, J. W. Hutchinson and V. S. Caviness, Mechanical model of brain convolutional development, Science 189(4196) (1975) 18-21.

66. J. Peng, J. Liu, B. Nie, Y. Li, B. Shan, G. Wang and K. Li, Cerebral and cerebellar gray matter reduction in first-episode patients with major depressive disorder: A voxel-based morphometry study, Eur. J. Radiol 80(2) (2011) 395-399.

67. W. B. Guo, F. Liu, Z. M. Xue, X. J. Xu, R. R. Wu, C. Q. Ma, S. C. Wooderson, C. L. Tan, X. L. Sun, J. D. Chen, Z. N. Liu, C. Q. Xiao, H. F. Chen and J. P. Zhao, Alterations of the amplitude of low-frequency fluctuations in treatment-resistant and treatment-response depression: A resting-state fMRI study, Prog. Neuro-Psychophol 37(1) (2012) $153-160$.

68. C. Ma, J. Ding, J. Li, W. Guo, Z. Long, F. Liu, Q. Gao, L. Zeng, J. Zhao and H. Chen, Restingstate functional connectivity bias of middle temporal gyrus and caudate with altered gray matter volume in major depression, Plos One 7(9) (2012) e45263.

69. Y. Zhang, C. Yu, Y. Zhou, K. Li, C. Li and T. Jiang, Decreased gyrification in major depressive disorder, Neuroreport 20(4) (2009) 378-380.

70. N. L. Nixon, P. F. Liddle, E. Nixon, G. Worwood, M. Liotti and L. Palaniyappan, Biological vulnerability to depression: Linked structural and functional brain network findings, Br. J. Psychiatry J. Mental Sci. 204 (2014) 283-289.
71. K. M. Han, E. Won, J. Kang, A. Kim, H. K. Yoon, H. S. Chang, K. R. Son, M. S. Lee, W. S. Tae and B. J. Ham, Local gyrification index in patients with major depressive disorder and its association with tryptophan hydroxylase-2 (TPH2) polymorphism, Hum Brain Mapp. 38(3) (2017) 1299-1310.

72. B. Cao, B. Mwangi, I. C. Passos, M. J. Wu, Z. Keser, G. B. Zunta-Soares, D. Xu, K. M. Hasan and J. C. Soares, Lifespan gyrification trajectories of human brain in healthy individuals and patients with major psychiatric disorders, Sci. Rep. 7(1) (2017) 511.

73. I. Fujita, The inferior temporal cortex: Architecture, computation and representation, J. Neurocytol. 31(3-5) (2002) 359-371.

74. A. Ishai, Let's face it: It's a cortical network, Neuroimage 40(2) (2008) 415-419.

75. J. A. Collins and I. R. Olson, Beyond the FFA: The role of the ventral anterior temporal lobes in face processing, Neuropsychol. 61 (2014) 65-79.

76. S. Amici, M. L. Gorno-Tempini, J. M. Ogar, N. F. Dronkers and B. L. Miller, An overview on primary progressive aphasia and its variants, Behav. Neurol. 17(2) (2006) 77-87.

77. M. L. Gorno-Tempini, N. F. Dronkers, K. P. Rankin, J. M. Ogar, L. Phengrasamy, H. J. Rosen, J. K. Johnson, M. W. Weiner and B. L. Miller, Cognition and anatomy in three variants of primary progressive aphasia, Ann. Neurol 55(3) (2004) 335-346.

78. W. W. Graves, R. Desai, C. Humphries, M. S. Seidenberg and J. R. Binder, Neural systems for reading aloud: A multiparametric approach, Cereb Cortex 20(8) (2010) 1799-1815.

79. J. Wang, L. Fan, Y. Zhang, Y. Liu, D. Jiang, Y. Zhang, C. Yu and T. Jiang, Tractography-based parcellation of the human left inferior parietal lobule, Neuroimage 63(2) (2012) 641-652.

80. J. J. Wang, S. M. Xie, X. Guo, B. Becker, P. T. Fox, S. B. Eickhoff and T. Z. Jiang, Correspondent functional topography of the human left inferior parietal lobule at rest and under task revealed using resting-state fMRI and coactivation based parcellation, Hum. Brain Mapp. 2017. 38(3) 1659 1675 .

81. J. E. Desmond, S. H. A. Chen, E. DeRosa, M. R. Pryor, A. Pfefferbaum and E. V. Sullivan, Increased frontocerebellar activation in alcoholics during verbal working memory: An fMRI study, Neuroimage 19(4) (2003) 1510-1520.

82. C. Ranganath, M. X. Cohen, C. Dam and M. D'Esposito, Inferior temporal, prefrontal and hippocampal contributions to visual working memory maintenance and associative memory retrieval, $J$. Neurosci. 24(16) (2004) 3917-3925.

83. W. J. Yang, Q. L. Chen, P. D. Liu, H. S. Cheng, Q. Cui, D. T. Wei, Q. L. Zhang and J. Qiu, Abnormal 
brain activation during directed forgetting of negative memory in depressed patients, J. Affect Disorders 190 (2016) 880-888.

84. A. I. Scott, R. H. Douglas, A. Whitfield and R. E. Kendell, Time course of cerebra; magnetic resonance changes after electroconvulsive therapy, Br. J. Psychiatry J. Mental Sci. 156 (1990) 551-553.

85. A. Yrondi, P. Peran, A. Sauvaget, L. Schmitt and C. Arbus, Structural-functional brain changes in depressed patients during and after electroconvulsive therapy, Acta neuropsychiatr. 30(1) (2018) 17-28.

86. M. Ahmadlou, H. Adeli and A. Adeli, Fractality analysis of frontal brain in major depressive disorder, Int. J. Psychophysiol. 85(2) (2012) 206-211.

87. M. Ahmadlou, H. Adeli and A. Adeli, Spatiotemporal analysis of relative convergence of EEGs reveals differences between brain dynamics of depressive women and men, Clinical EEG Neurosci. 44(3) (2013) 175-181.

88. U. R. Acharya, V. K. Sudarshan, H. Adeli, J. Santhosh, J. E. Koh and A. Adeli, Computer-Aided Diagnosis of Depression Using EEG Signals, Eur. Neurol. 73(5-6) (2015) 329-336.

89. U. R. Acharya, V. K. Sudarshan, H. Adeli, J. Santhosh, J. E. Koh, S. D. Puthankatti and A. Adeli, A novel depression diagnosis index using nonlinear features in EEG signals, Eur. Neurol. 74(1-2) (2015) 79-83.

90. U. R. Acharya, S. L. Oh, Y. Hagiwara, J. H. Tan, H. Adeli and D. P. Subha, Automated EEG-based screening of depression using deep convolutional neural network, Comput. Methods Prog. Biomed. 161 (2018) 103-113. 